\title{
Manipulation of pain catastrophizing: An experimental study of healthy participants
}

\author{
Joel E Bialosky ${ }^{\text {* }}$ \\ Adam T Hirsh ${ }^{2,3}$ \\ Michael E Robinson ${ }^{2,3}$ \\ Steven Z George ${ }^{1,3 *}$ \\ 'Department of Physical Therapy; \\ ${ }^{2}$ Department of Clinical and Health \\ Psychology; ${ }^{3}$ Center for Pain Research \\ and Behavioral Health, University of \\ Florida, Gainesville, Florida, USA
}

\begin{abstract}
Pain catastrophizing is associated with the pain experience; however, causation has not been established. Studies which specifically manipulate catastrophizing are necessary to establish causation. The present study enrolled 100 healthy individuals. Participants were randomly assigned to repeat a positive, neutral, or one of three catastrophizing statements during a cold pressor task (CPT). Outcome measures of pain tolerance and pain intensity were recorded. No change was noted in catastrophizing immediately following the CPT $\left(\mathrm{F}_{(1,84)}=0.10, \mathrm{p}=0.75\right.$, partial $\left.\eta^{2}<0.01\right)$ independent of group assignment $\left(F_{(4,84)}=0.78, p=0.54\right.$, partial $\left.\eta^{2}=0.04\right)$. Pain tolerance $\left(\mathrm{F}_{(4)}=0.67, \mathrm{p}=0.62\right.$, partial $\left.\eta^{2}=0.03\right)$ and pain intensity $\left(\mathrm{F}_{(4)}=0.73, \mathrm{p}=0.58\right.$, partial $\eta^{2}=0.03$ ) did not differ by group. This study suggests catastrophizing may be difficult to manipulate through experimental pain procedures and repetition of specific catastrophizing statements was not sufficient to change levels of catastrophizing. Additionally, pain tolerance and pain intensity did not differ by group assignment. This study has implications for future studies attempting to experimentally manipulate pain catastrophizing.
\end{abstract}

Keywords: pain, catastrophizing, experimental, cold pressor task, pain catastrophizing scale
Correspondence: Steven Z George Health Science Center, PO Box 100154 , Gainesville, FL 32610-0154, USA

Tel +l 3522736432

Fax +I 3522736109

Email szgeorge@phhp.ufl.edu
Pain catastrophizing is "an exaggerated negative orientation toward noxious stimuli" (Sullivan et al 1995) and is associated with heightened pain response in both clinical (Severeijns et al 2001; Tan et al 2001) and experimental (Geisser et al 1992; Sullivan et al 1995; Osman et al 1997) pain studies. For example, catastrophizing has been associated with pain intensity and disability in individuals with chronic pain (Severeijns et al 2001; Peters et al 2005). Additionally, catastrophizing is associated with a heightened pain response during a cold pressor task (CPT) in healthy individuals (Sullivan et al 1995). Although these studies demonstrate a robust association between catastrophizing and pain, their cross-sectional nature limits interpretations regarding causation.

Prospective studies indicate a temporal association between catastrophizing and pain and are more suggestive of causation. For example, preoperative measures of catastrophizing are predictive of postoperative pain intensity (Granot and Ferber 2005; Pavlin et al 2005), and catastrophizing measured during acute dental pain is predictive of thermal pain threshold and tolerance upon resolution of the dental pain (Edwards et al 2004).

A limitation of the current literature is the lack of studies which specifically manipulate catastrophizing to determine its effect on pain. Such study designs are necessary in order to strengthen conclusions about causation. To our knowledge, only one prior study has attempted to manipulate catastrophizing during an experimental pain task (Severeijns et al 2005). Severeijns and colleagues (2005) manipulated catastrophizing by increasing the threat of the stimulus (ie, a risk of passing out during a CPT) for healthy participants. A small increase in catastrophizing was noted following 
the instructional set; however, no group differences in pain intensity or tolerance were noted during the CPT.

A potential limitation of the study by Severeijns and colleagues (2005) was the attempt to manipulate catastrophizing through exaggerated threat level. Catastrophizing is comprised of cognitions related to rumination, helplessness, and magnification (Sullivan et al 1995, 2001; Osman et al 1997; Van Damme et al 2002). These cognitions were not specifically manipulated in the Severeijns and colleagues (2005) study. Experimental manipulation of catatastrophizing may require attention to these specific cognitions in order to meaningfully alter catastrophizing and influence subsequent pain responses. We are unaware of any prior studies which have adopted such methodology to study the effect of catastrophizing on pain.

Therefore, the current study had two purposes. The first was to determine whether pain catastrophizing could be successfully manipulated through the repetition of rumination, helplessness, and magnification catastrophizing statements. We hypothesized that individuals repeating statements consistent with the construct of catastrophizing would have higher scores on measures of pain catastrophizing immediately following a CPT than those repeating a positive and neutral statement. The second purpose was to assess the effect of rumination, helplessness, and magnification statements on pain intensity and tolerance during a CPT. We hypothesized that repeating a pain catastrophizing statement would result in higher ratings of pain intensity and lower tolerance to a CPT.

\section{Methods}

\section{Participants}

The present study was approved by the University of Florida Institutional Review Board. Participants between the ages of 18 and 25 were recruited from the University of Florida Health Science Center by flyers and word of mouth. Individuals currently experiencing pain or taking pain medication were excluded, as were non-English speaking individuals. Participants meeting the inclusion/ exclusion criteria and agreeing to participate signed an informed consent form and completed the following questionnaires.

\section{Measures}

\section{Demographics form}

Information was obtained pertaining to the participants' sex, age, ethnicity, educational level, and prior experience with the CPT.

\section{Pain catastrophizing scale}

The Pain Catastrophizing Scale (PCS) (Sullivan et al 1995) is comprised of 13 items specific to coping with pain and makes use of a five point ordinal scale from 0 to 4 . Subjects are asked to quantify each statement in terms of its applicability towards a previous painful episode, with higher scores indicating a greater level of catastrophizing. A total score and three subscale scores consisting of rumination, magnification, and helplessness, may be calculated. Prior studies have validated the factor structure and found good internal consistency, reliability, and validity (Sullivan et al 1995; Osman et al 1997; Van Damme et al 2002).

\section{Fear of pain questionnaire (FQP-III)}

The FPQ-III (McNeil and Rainwater 1998) consists of 30 items, each scored on a 5-point adjectival scale, which measures fear of normally painful situations. Higher scores indicate greater pain related fear. The FPQ has demonstrated sound psychometric properties in both experimental and clinical pain studies (McNeil and Rainwater 1998; Osman et al 2002; Roelofs et al 2005). Fear of pain has been previously shown to influence CPT pain (Keogh et al 2003; Sullivan et al 2004; George et al 2006) and we wished to be certain our randomization process was successful in preventing group differences in baseline fear of pain.

\section{Group assignment}

Participants were randomly assigned to repeat one of five statements during the CPT. The catastrophizing statements were taken directly from the PCS and selected based on the strength of their factor loading to each respective construct (Sullivan et al 1995).

Pain Catastrophizing Group 1: Received a statement consistent with magnification and were instructed to repeat the statement, "I fear the pain will get worse."

Pain Catastrophizing Group 2: Received a statement consistent with helplessness and were instructed to repeat the statement, "I fear I can't go on."

Pain Catastrophizing Group 3: Received a statement consistent with rumination and were instructed to repeat the statement, "I keep thinking how badly I want the pain to stop."

Positive Statement Group: This group was instructed to repeat the statement, "I can overcome the pain."

Neutral Statement Group: This group was instructed to repeat the statement, "The sky is blue." 


\section{Procedure}

Participants provided informed consent and then completed the demographic form, the PCS, and the FPQ-III. Next, participants were instructed in the use of the Visual Analog Scale (VAS). The VAS consists of a horizontal $10 \mathrm{~cm}$ line anchored by "no pain" and "worst pain imaginable." Participants were instructed to make a vertical mark along the horizontal line to indicate their pain rating during the study whenever a VAS was placed in front of them. VASs of pain are significantly correlated with other measures of pain and have been widely used in experimental pain studies (Jensen et al 1986; Good et al 2001). Participants were asked to mark their baseline rating of pain on a VAS prior to the CPT to ensure understanding of the use of the VAS and that they were currently pain free. Participants were then randomly assigned to repeat one of the five statements aloud during the CPT. The assigned statement was then fixed to the wall in front of the participant who was asked to read the statement aloud two times and instructed that they would continually repeat the statement aloud for the duration of the CPT. The following statement was then provided regarding the cold pressor.

"I will ask you to submerge your NON-DOMINANT hand, up to your wrist, into this container of water. You can remove your hand from the water when you can no longer tolerate the pain, but it is important that you leave your hand in the water as long as you possibly can. Do you understand what to do?"

Upon verbalization of understanding of the study protocol, participants were instructed to place their hand into the cold pressor. The CPT consisted of a circulating water bath maintained at $2{ }^{\circ} \mathrm{C}\left( \pm 0.5^{\circ} \mathrm{C}\right)$. Participants repeated their assigned statement aloud beginning immediately upon placement of their hand into the cold pressor. Pain ratings were recorded via the VAS at the onset of pain and every 15 seconds following the initiation of the CPT. The examiner indicated the need to complete a VAS by placing a clean one in front of the participant every fifteen seconds from the initiation of the CPT and when the participant withdrew from the CPT. Completed VASs were immediately removed by the researcher and out of sight of participants completing subsequent VASs. Participants not removing their hand from the CPT by 3 minutes were instructed to remove their hand by the investigator. Following the CPT, participants again completed the PCS with instruction to have responses reflect what they were experiencing during the CPT.

\section{Data analysis}

Descriptive statistics were generated for continuous and categorical measures. Univariate ANOVA was used to assess post-randomization differences in continuous variables of demographic and psychological measures. Chi-square analysis was used to assess post-randomization group differences in categorical demographic variables.

First, we used Pearson correlation coefficients to examine whether an association existed between both pre and post CPT measures of pain catastrophizing and pain intensity and tolerance. Next we analyzed pain catastrophizing and whether this was influenced by group assignment using a $2 \times 5$ repeated measure ANOVA. PCS score at baseline and immediately following the CPT served as the within subject factor while group assignment was the between subject factor. Post hoc testing using Bonferroni correction was performed as indicated by the ANOVA model.

Individual univariate ANOVA models were used to assess the effect of group assignment on both pain tolerance and rating of pain intensity provided by the participant at the time of withdrawal from the CPT (tolerance), with post hoc testing using Bonferroni correction as indicated by the ANOVA model. Finally, repeated measure ANOVA was used to test the effect of group assignment on pain perception at fifteen second increments from baseline to one minute. This exploratory analysis was performed only on subjects that tolerated the CPT for one minute. Its purpose was to determine if there were any group differences in pain intensity ratings before tolerance because we were concerned that a ceiling effect might exist for pain intensity ratings at tolerance.

\section{Results}

100 subjects met the criteria and consented to participate in the study. No baseline differences were observed between the groups in demographic characteristics; however, fear of pain approached significance (Table 1), so the decision was made to include this as a covariate in subsequent analysis. Pre CPT PCS scores were not significantly correlated with pain intensity $(r=0.05, p=0.59)$ or tolerance $(r=-0.12, p=0.25)$. Furthermore, post CPT PCS scores were not significantly correlated with pain intensity $(\mathrm{r}=0.12, \mathrm{p}=0.25)$; however, a significant correlation was observed between post CPT PCS scores and pain tolerance $(r=-0.31, p<0.01)$.

\section{Purpose I: Effect of coping statement on self-report of pain catastrophizing}

In the model without controlling for fear of pain, a group $\times$ time (pre to post CPT) interaction for change in PCS scores was not present $\left(\mathrm{F}_{(4,95)}=0.94, \mathrm{p}=0.45\right.$, partial $\left.\eta^{2}=0.04\right)$; however, a main effect for assessment time was observed $\left(\mathrm{F}_{(1,95)}=5.52, \mathrm{p}=0.02\right.$, partial $\eta^{2}=0.06$ ), with higher PCS scores following the CPT 
Table I Baseline characteristics of the individual instructional set intervention groups

\begin{tabular}{|c|c|c|c|c|c|c|c|}
\hline & Magnification & Helplessness & Rumination & Positive & Neutral & Total & p- value \\
\hline \multicolumn{8}{|l|}{ Sex: } \\
\hline Male & 6 & 8 & 7 & 4 & 9 & 34 & \\
\hline Female & 14 & 11 & 13 & 17 & 11 & 66 & 0.42 \\
\hline Age: (years, SD) & $20.80(1.70)$ & $21.53(1.39)$ & $21.45(1.88)$ & $21.10(1.97)$ & $21.10(1.74)$ & $21.19(1.74)$ & 0.69 \\
\hline \multicolumn{8}{|l|}{ Race: } \\
\hline Caucasian & 10 & 9 & 11 & 7 & 9 & 46 & \\
\hline \multicolumn{8}{|l|}{ African } \\
\hline American & 3 & 2 & 0 & 2 & 3 & 10 & \\
\hline Other & 7 & 8 & 9 & 12 & 8 & 44 & 0.93 \\
\hline Education: (years, SD) & I5.20 (I.64) & I5.53 (I.7I) & $14.85(1.27)$ & $15.10(1.61)$ & $15.15(1.66)$ & $15.16(1.57)$ & 0.77 \\
\hline \multicolumn{8}{|l|}{ Prior CP Experience: } \\
\hline Yes & 2 & 7 & 5 & 5 & 7 & 26 & \\
\hline No & 18 & 12 & 15 & 15 & 13 & 73 & 0.44 \\
\hline PCS (SD) & $18.45(10.16)$ & $15.32(7.65)$ & $22.20(8.92)$ & |8.7| (8.79) & $18.00(9.70)$ & 18.57 (9.17) & 0.23 \\
\hline FPQ (SD) & $46.00(14.23)$ & $40.54(19.00)$ & $53.85(17.04)$ & $56.68(19.14)$ & $51.67(14.55)$ & $50.34(17.28)$ & 0.06 \\
\hline
\end{tabular}

Notes: Baseline characteristics of the group assignments for catastrophizing statements and a neutral statement. P was set at significant at $<0.05$. Abbreviations: $C P$, cold pressor; FPQ, fear of pain questionnaire; PCS, pain catastrophizing scale; SD, standard deviation.

(mean difference 2.00, $\mathrm{SD}=8.49, \mathrm{p}=0.02$, effect size $=0.24$ ). When fear of pain was included in the model as a covariate (due to potential post randomization differences), neither a group $\times$ time (pre- to post-CPT) interaction $\left(\mathrm{F}_{(4,84)}=0.78\right.$, $\mathrm{p}=0.54$, partial $\left.\eta^{2}=0.04\right)$; nor a main effect for assessment time $\left(\mathrm{F}_{(1,84)}=0.10, \mathrm{p}=0.75\right.$, partial $\left.\eta^{2}<0.01\right)$ was observed for change in PCS scores. Due to the lack of a group effect for total PCS scores, we further investigated whether group assignment had a specific effect on individual constructs of the PCS. For example, we considered whether change in rumination was observed only for those subjects repeating rumination statements. Group x time interactions were not observed for any of these comparisons that considered individual PCS constructs $(\mathrm{p}>0.05)$.

\section{Purpose 2: Effect of group assignment on tolerance and pain intensity}

In the model with fear of pain as a covariate (due to potential post randomization differences), no significant group differences were observed in tolerance $\left(\mathrm{F}_{(4)}=0.67, \mathrm{p}=0.62\right.$, partial $\eta^{2}=0.03$ ) (Figure 1 ) or pain intensity rating provided by the participant at the time of withdrawal from the CPT (tolerance) $\left(\mathrm{F}_{(4)}=0.73, \mathrm{p}=0.58\right.$, partial $\left.\eta^{2}=0.03\right)$. Subsequently, we explored group differences in pain intensity at earlier immersion times as previously indicated (Figure 2). Forty five participants maintained their hand in the CPT for a minimum of one minute and were included in this analysis. A main effect for time was present $\left(\mathrm{F}_{(4,36)}=4.21, \mathrm{p}=0.01\right.$, partial $\left.\eta^{2}=0.32\right)$ suggesting a general increase in pain over time of immersion; however, similar to our findings at tolerance, no group differences occurred in pain intensity ratings across any of the time points $\left(\mathrm{F}_{(16,156)}=0.42, \mathrm{p}=0.98\right.$, partial $\left.\eta^{2}=0.04\right)$. Our findings for tolerance and pain intensity did not differ when fear of pain was removed from the model or when the three individual catastrophizing groups were collapsed to a single catastrophizing group.

\section{Discussion}

Studies which specifically manipulate catastrophizing prior to measuring pain are lacking from the literature and are necessary to establish causation. Similar to prior studies (Geisser et al 1992; Sullivan et al 1995; Osman et al 1997), catastrophizing in our study was associated with experimental pain response. Specifically, subjects with higher ratings of post CPT catastrophizing demonstrated decreased tolerance to the CPT. The association between catastrophizing and pain has been found to vary depending on when catastrophizing is measured. For example, catastrophizing measured immediately following experimental pain has been found to correlate more strongly to pain tolerance and severity than measures taken prior to the experimental pain experience (Dixon et al 2004; Edwards et al 2005). Our findings were similar in that only post experimental pain measures of catastrophizing correlated significantly with pain tolerance.

We attempted to manipulate catastrophizing through the repetition of statements directly related to the specific 


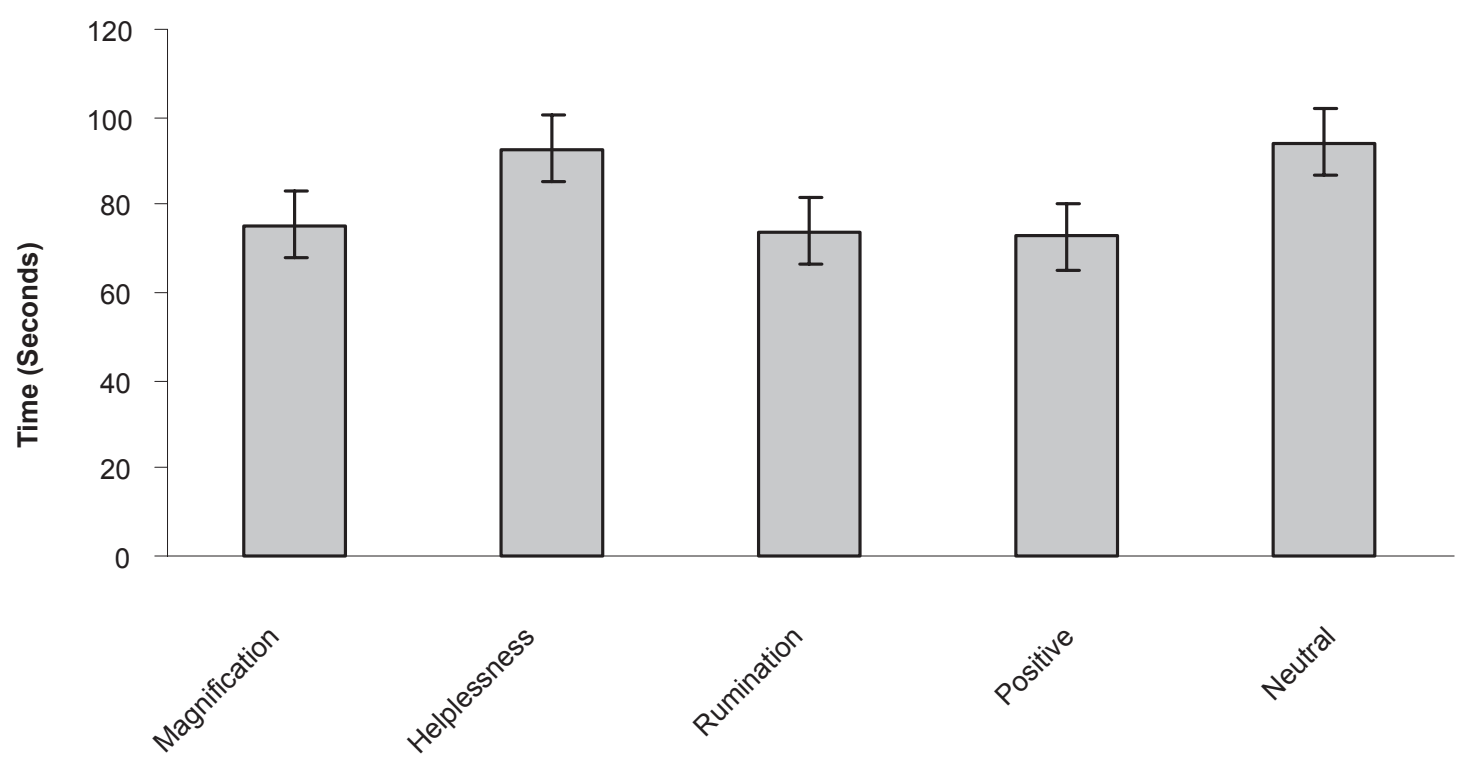

Figure I Tolerance to cold-pressor.

A significant difference $(p>0.05)$ in tolerance to the cold- pressor task was not present between the participants repeating a catastophizing statement and those repeating a neutral statement. Error bars represent one standard error of the mean.

constructs of rumination, magnification, and helplessness. Catastrophizing ratings following the CPT did not differ from ratings obtained prior to the CPT task. Our findings differ from Severeijns and colleagues (2005) who noted a significant, albeit small, increase in catastrophizing following experimental manipulation. The present study and the small changes observed by Severeijns and colleagues (2005) suggest that catastrophzing may be difficult to manipulate and changes which do occur may be small in magnitude.

Methodological differences may explain the small but significant group dependent changes in catastrophizing observed by Severeijns and colleagues (2005) which were not present in our study. Specifically, threat has been linked to pain catastrophizing in experimental pain

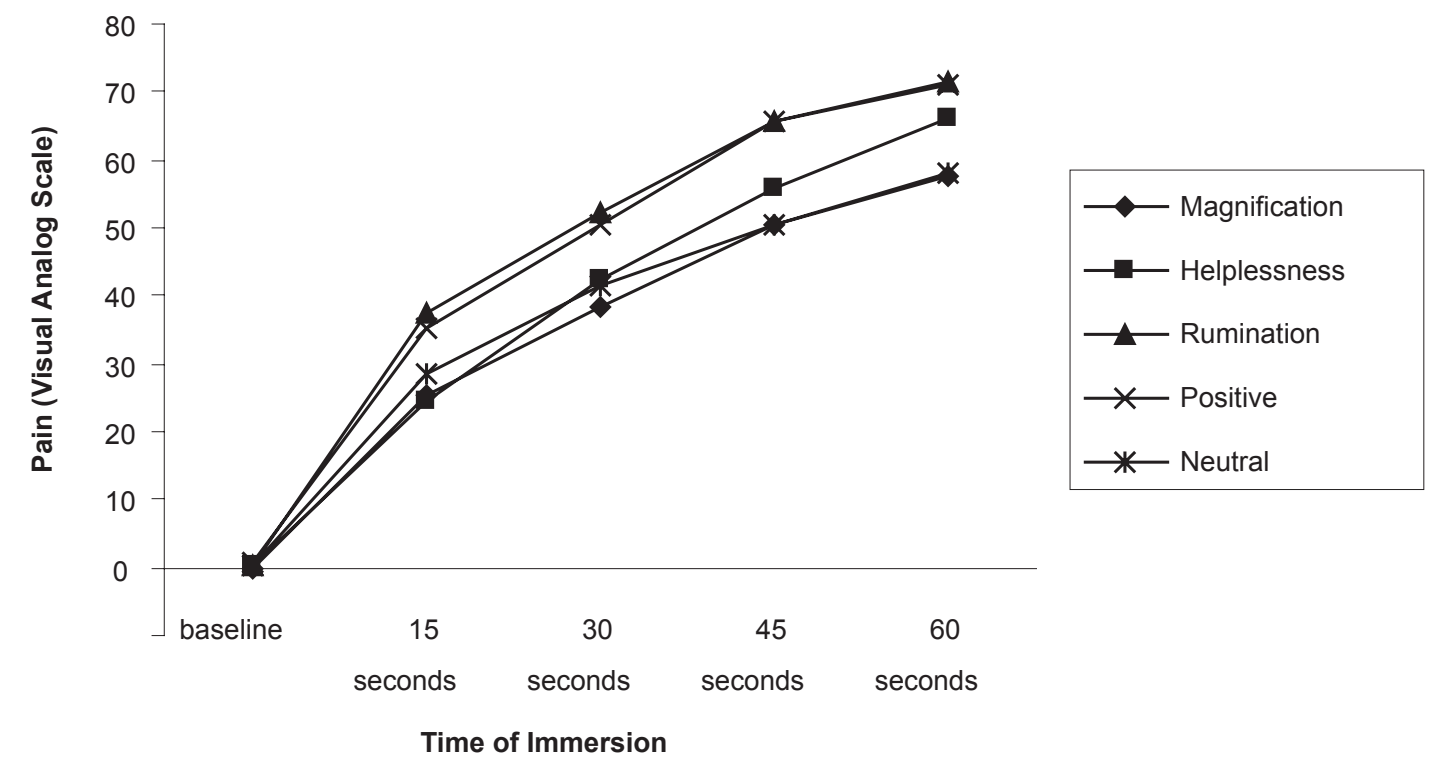

Figure 2 Self report of pain throughout the cold pressor task.

Notes: A significant difference $(p>0.05)$ in pain intensity did not exist between the participants repeating a catastophizing statement and those repeating a neutral statement. 
(Jackson et al 2005), and Severeijns and colleagues (2005) manipulated catastrophizing specifically through the increase of threat level. Our attempts to experimentally manipulate catastrophizing may have been ineffective due to an inadequate threat presented by the CPT to healthy participants. Subsequently, group assignment may have been inadequate to alter baseline level of catastrophizing beyond the increase associated with exposure to the experimental pain. Greater and group specific changes in catastrophizing may have occurred had we manipulated the threat level of the CPT along with the specific catastrophizing statement. We are unable offer more than speculation on this point as we did not measure threat level in this study.

Similar to Severeijns and colleagues (2005), we did not observe group differences in pain tolerance or intensity. While studies have found a temporal relationship between catastrophizing and the pain experience (Geisser et al 1992; Sullivan et al 1995; Edwards et al 1994; Granot and Ferber 2005), neither our study nor that of Severeijns and colleagues (2005) was able to affect the pain experience with attempted direct manipulation of catastrophizing. A plausible explanation is that neither study adequately manipulated catastrophizing. Experimentally induced pain in otherwise healthy participants may be an inadequate method to study this phenomenon due to brief stimuli with known ending parameters. An additional explanation is the possibility pain catastrophzing may be a trait and not subject to manipulation (Sullivan et al 2001). While a consensus has not been reached, catastrophizing has demonstrated change in response to therapeutic interventions suggesting a state like characteristic amenable to manipulation (Moss-Morris et al 2007; Thorn et al 2007; Voerman et al 2007; Vowles et al 2007).

Limitations of this study include a healthy sample which may not be generalizable to people experiencing pain. Future studies may wish to attempt to manipulate catastrophizing in individuals experiencing clinical pain or using a more ecologically valid pain stimulus such as delayed onset muscle soreness. A second limitation of the study is the disproportionate number of women to men. Sex differences have been observed in pain catastrophizing (Sullivan et al 1995; Osman et al 1997) and may confound the ability to manipulate catastrophizing. Unfortunately, our sample size was not adequate to test for the influence of sex on the studied outcomes. A third limitation of the current study is the selection of statements for group assignment based on previously reported factor structure of the PCS and other commonly used statements associated with positive and neutral coping. Differences in the complexity of the statements, as well as the focus of the statements could have influenced the results. Specifically differences existed in the length of the individual statements and the catastrophizing statement associated with rumination had a cognitive focus, while the other catastrophizing statements had an affective focus. Prior studies have observed sex differences in coping strategies for pain (Affleck et al 1999; Keogh and Herdenfeldt 2002; Keogh et al 2005). Specifically, women are more likely to use emotion-focused strategies (Affleck et al 1999) and manipulation of coping strategies may have differing effects by sex (Keogh and Herdenfeldt 2002; Keogh et al 2005). The attempted manipulation of pain catastrophizing could plausibly differ by the focus (cognitive or affective) of the intervention and the sex of the individual participant. Finally, we did not perform a specific power analysis as we were uncertain as to the magnitude of the effect of catastrophizing. We chose our initial sample size as 100 with the expectation that this would allow us to detect a difference with an effect size between 0.3 and 0.4 . A post hoc power analysis for our study shows a power of $31 \%$; however, based on the small effect sizes, we believe that our findings are suggestive of little to no effect rather than the study being underpowered to find such an effect.

Despite the limitations, the present study offers important methodological considerations for the design of future studies. First, the repetition of phrases consistent with magnification, helplessness, and rumination did not significantly alter pain catastrophizing in comparison to neutral or positive phrases. Based on a prior study (Severeijn et al 2005), manipulation of catastrophizing through threat level of an experimental pain procedure may be a better way to manipulate pain catastrophizing. Second, consistent with prior studies (Dixon et al 2004; Edwards et al 2005), measures of pain catastrophizing taken immediately following an experimental pain task better correlated to experimental pain outcomes. Future studies attempting to experimentally manipulate pain catastrophizing may wish to take baseline measures immediately following an experimental pain task and assess changes in catastrophizing associated with experimental manipulation in reference to this. Finally, the present study failed to manipulate catastrophizing and the magnitude of change reported in a prior study which did successfully manipulate catastrophizing (Severeijn et al 2005) was small. Subsequently, the CPT with or without altered threat level may be an ineffective method of experimental pain to test the clinically meaningful manipulation of pain catastrophizing in healthy individuals. 
Future studies may wish to use alternative methods of experimental pain to determine if catastrophizing can be manipulated to a greater magnitude.

\section{Conclusion}

Catastrophizing is associated with the pain experience; however, causation has not been established. We attempted to experimentally manipulate catastrophizing in healthy individuals to observe the effect on pain tolerance and intensity. We did not observe a change in pain catastrophizing following attempted manipulation. Furthermore, group differences did not emerge for pain tolerance or intensity. This study is one of the first to attempt to experimentally manipulate pain catastrophizing and offers important methodological considerations for future research.

\section{Acknowledgments}

JEB received support from the NIH T-32 Neural Plasticity Research Training Fellowship (T32HD043730). SZG (PI) and MER received support from a grant from NIH-NIAMS (AR051128) while preparing this manuscript. Ryland Galmish assisted with recruitment and data collection.

\section{References}

Affleck G, Tennen H, Keefe FJ, et al. 1999. Everyday life with osteoarthritis or rheumatoid arthritis: independent effects of disease and gender on daily pain, mood, and coping. Pain, 83:601-9.

Dixon KE, Thorn BE, Ward LC. 2004. An evaluation of sex differences in psychological and physiological responses to experimentally-induced pain: a path analytic description. Pain, 112:188-96.

Edwards RR, Campbell CM, Fillingim RB. 2005. Catastrophizing and experimental pain sensitivity: only in vivo reports of catastrophic cognitions correlate with pain responses. J Pain, 6:338-9.

Edwards RR, Fillingim RB, Maixner W, et al. 2004. Catastrophizing predicts changes in thermal pain responses after resolution of acute dental pain. J Pain, 5:164-70.

Geisser M, Robinson M, Pickern W. 1992. Differences in cognitive coping strategies among pain-sensitive and pain-tolerant individuals on the cold-pressor test. Behav Ther, 23:31-41.

George SZ, Dannecker EA, Robinson ME. 2006. Fear of pain, not pain catastrophizing, predicts acute pain intensity, but neither factor predicts tolerance or blood pressure reactivity: an experimental investigation in pain-free individuals. Eur J Pain, 10:457-65.

Good M, Stiller C, Zauszniewski JA, et al. 2001. Sensation and Distress of Pain Scales: reliability, validity, and sensitivity. J Nurs Meas, 9:219-38.

Granot M, Ferber SG. 2005. The roles of pain catastrophizing and anxiety in the prediction of postoperative pain intensity: a prospective study. Clin J Pain, 21:439-45.

Jackson T, Pope L, Nagasaka T, et al. 2005. The impact of threatening information about pain on coping and pain tolerance. $\mathrm{Br} J$ Health Psychol, 10(Pt 3):441-51.
Jensen MP, Karoly P, Braver S. 1986. The measurement of clinical pain intensity: a comparison of six methods. Pain, 27:117-26.

Keogh E, Bond FW, Hanmer R, et al. 2005. Comparing acceptance- and control-based coping instructions on the cold-pressor pain experiences of healthy men and women. Eur J Pain, 9:591-8.

Keogh E, Herdenfeldt M. 2002. Gender, coping and the perception of pain. Pain, 97:195-201.

Keogh E, Thompson T, Hannent I. 2003. Selective attentional bias, conscious awareness and the fear of pain. Pain, 104:85-91.

McNeil DW, Rainwater AJ, III. 1998. Development of the Fear of Pain Questionnaire - III. J Behav Med, 21:389-410.

Moss-Morris R, Humphrey K, Johnson MH, et al. 2007. Patients' perceptions of their pain condition across a multidisciplinary pain management program: do they change and if so does it matter? Clin J Pain, 23:558-64.

Osman A, Barrios FX, Kopper BA, et al. 1997. Factor structure, reliability, and validity of the Pain Catastrophizing Scale. J Behav Med, 20:589-605.

Osman A, Breitenstein JL, Barrios FX, et al. 2002. The Fear of Pain Questionnaire-III: further reliability and validity with nonclinical samples. J Behav Med, 25:155-73.

Pavlin DJ, Sullivan MJ, Freund PR, et al. 2005. Catastrophizing: a risk factor for postsurgical pain. Clin J Pain, 21:83-90.

Peters ML, Vlaeyen JW, Weber WE. 2005. The joint contribution of physical pathology, pain-related fear and catastrophizing to chronic back pain disability. Pain, 113:45-50.

Roelofs J, Peters ML, Deutz J, et al. 2005. The Fear of Pain Questionnaire (FPQ): further psychometric examination in a non-clinical sample. Pain, 116:339-46.

Severeijns R, van den Hout MA, Vlaeyen JW. 2005. The causal status of pain catastrophizing: an experimental test with healthy participants. Eur J Pain, 9:257-65.

Severeijns R, Vlaeyen JW, van den Hout MA, et al. 2001. Pain catastrophizing predicts pain intensity, disability, and psychological distress independent of the level of physical impairment. Clin J Pain, 17:165-72.

Sullivan MJ, Bishop S, Pivik J. 1995. The Pain Catastrophizing Scale: Development and Validation. Psychol Asess, 4:524-32.

Sullivan MJ, Thorn B, Haythornthwaite JA, et al. 2001. Theoretical perspectives on the relation between catastrophizing and pain. Clin $J$ Pain, 17:52-64.

Sullivan MJ, Thorn B, Rodgers W, et al. 2004. Path model of psychological antecedents to pain experience: experimental and clinical findings. Clin J Pain, 20:164-73.

Tan G, Jensen MP, Robinson-Whelen S, et al. 2001. Thornby JI, Monga TN. Coping with chronic pain: a comparison of two measures. Pain, 90:127-33.

Thorn BE, Pence LB, Ward LC, et al. 2007. A randomized clinical trial of targeted cognitive behavioral treatment to reduce catastrophizing in chronic headache sufferers. J Pain, 8:938-49.

Van Damme S, Crombez G, Bijttebier P, et al. 2002. A confirmatory factor analysis of the Pain Catastrophizing Scale: invariant factor structure across clinical and non-clinical populations. Pain, 96:319-24.

Voerman GE, Sandsjo L, Vollenbroek-Hutten MM, et al. 2007. Changes in cognitive-behavioral factors and muscle activation patterns after interventions for work-related neck-shoulder complaints: relations with discomfort and disability. J Occup Rehabil, 17:593-609.

Vowles KE, McCracken LM, Eccleston C. 2007. Processes of change in treatment for chronic pain: the contributions of pain, acceptance, and catastrophizing. Eur J Pain, 11:779-87. 
